\title{
用全息相祄干涉显微术定量研究 晶一液界面的溶质边界层*
}

于锡玲 岳学锋 高航军 任红文

(山东大学晶体材料研㠰所, 济南 250100)

关锤词全息术、边界层

\section{一、引 言}

溶液生长晶体, 在晶一液界面附近, 有关质量输运的真实行为, 至今还是一个有待研究的课 题。近几年有不少文献报道, 采用全息干涉术研究晶体周围的浓度场分布或测量浓度边界层 的厚度 ${ }^{[1-4]}$. 然而这些研究均是在自然对流的状态下, 进行总体分析. 而对输运的重要地 带一一边界层内的溶质分布, 或在强迫对流状态下, 层内的变化规律未见报道. 另外对检测方 法的准确度也末见讨论.

本文报道一种有效的实时测量技术一一全息相衬干涉显微木。用该支术对溶液不同对流 状态下, 晶一液界面的溶质边界层厚度和层内浓度分布进行了检测, 并给出了精确测量的必要 条件.

\section{二、全息相祄干涉显微术}

1. 基本原理 用全息相衬显微术研究晶体生长的基本原理作者已在文献 [5] 中给 出. 将它与全息干涉原理相结合,即获得全息相祄干涉显微术. 也就是说,将滤波后的物光在两个 不同时刻进行比较. 由于晶体生长引起了光的位相改变,当位相差满足下式

$$
\Delta \phi=(2 K+1) \Pi
$$

时, 就会产生千涉暗条纹, 式中 $|K|=0,1,2, \cdots \cdots$. 将此干涉图置于显微镜下就可观察和 计量局部的细微构造。

\section{2. 精确测典洝质边界层的必要杂件}

1) 晶体生长是各向异性界面向三维空间伸展的过程, 各晶面周围的环境是不相同的. 要 研究某一晶面的环境, 就必须使该晶面的环境相充分显露. 其简单有效的方法是让该晶面完 全平行于光轴.

2）欲精确测量干涉条级的位置和边界层的厚度，就必须精确定位全息像接收屏的位置， 也即只有在透镜成象的象平面上才能获得最佳的信息. 通常既要保证被测晶面完全平行于光 轴, 又要精确定位象面位置, 在实验上是不易做到的, 而采用相祄显微术即可方便地达到目 的.

本文 1989 年 2 月 27 日收到. 1989 年 8 月 25 日收到改稿。

- 国家自然科学基金资助项目. 
因为任何物体的边缘当光照射时都产生衍射条纹.这些衍射条纹在相衬显微镜下清晰可 见. 如果接收屏离开象面,棱边的衍射条纹都要增多同时加宽,而且以象面的位置为原点, 以 校边为分界线进行方位更换。只有在象面上,棱边衍射条纹少而细,同时晶体形貌行射象的清 晰度最佳。因此,利用棱边衍射现象作为判据,定位全息象接收屏既精确，又方便。同时有助 于校正被测晶面是否平行于光轴。

3）灿果被测晶体尺寸较大或生长液的过饱和度较大时,应注意光线的折射和端部效应造 成的影响. 因为干涉方程假定光线是直射，而实际上光线经过折射率梯度较大的区域,光的折 射造成的偏折是不可忽略的. 其次光线通过边界层的人射端和出射端是非二维性问题，所以 精确测量要做偏折误差及端部误差的修正 ${ }^{[6]}$.

\section{三、溶质边界层的测是}

1. 架检方法 光源为 $\mathrm{He}-\mathrm{Ne}$ 激光器, 功率为 $25 \mathrm{~mW}$. 育晶器的温度由超级恒温槽控 制, 精度为 $\pm 0.05^{\circ} \mathrm{C}$. 溶液体积为 $250 \mathrm{ml}$, 由电磁搅拌器使其强迫对流. 磷酸二氢钾 (KDP) 晶体尺寸为 $6 \times 6 \times 10 \mathrm{~mm}$ 左右. $\mathrm{KDP}$ 饱和溶液 $\left(31.9^{\circ} \mathrm{C}\right)$ 在时刻 $\imath_{1}$ 曝光一次记录的全息图 经处理后,再把晶体放人溶液,采用相村显微术调准定位. 通过温度的改变, 可获得过饱和度 不同的溶质边界层的全息图,见图 1 .
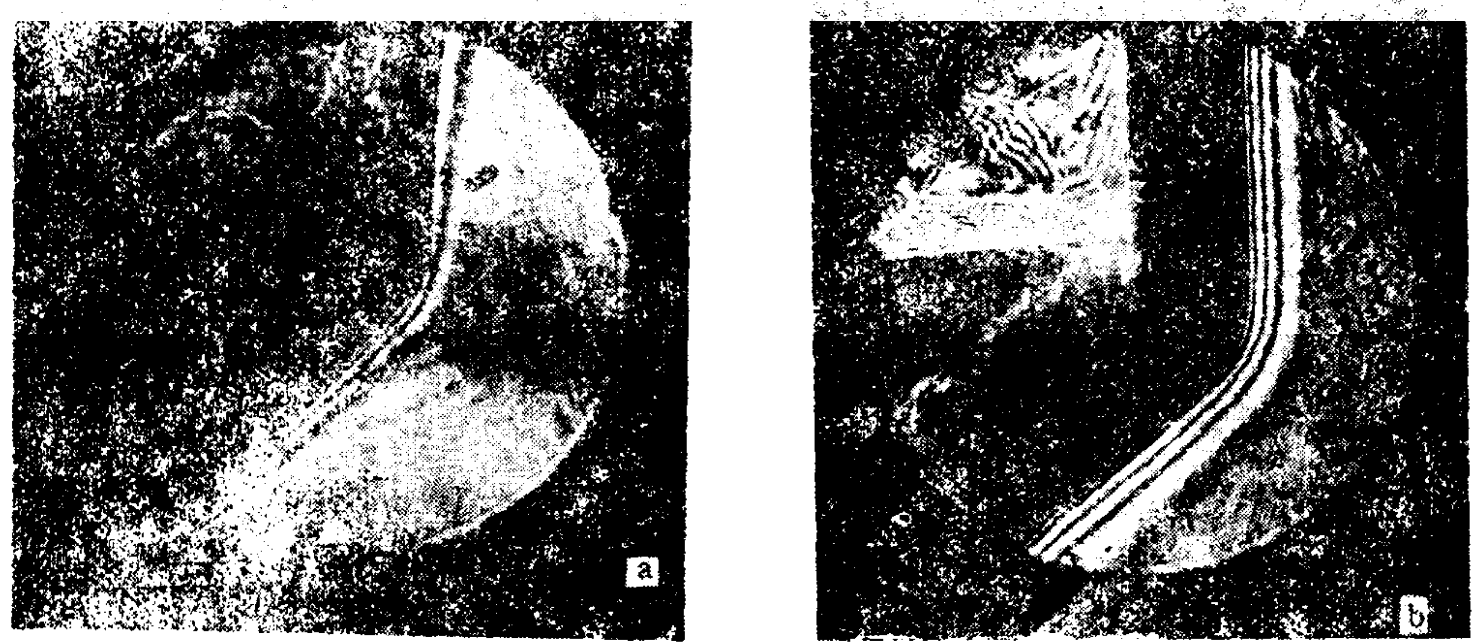

图 $1 \mathrm{KDP}$ 溶液的过饱和度不同时, 边界层不同的全息图 a. 晶体生长 $\Delta C=1.04 \% ; \mathrm{b}$ 溶解 $\Delta C=-0.19 \%$

若在相同的过饱和度下,改变溶液的运动状态,即可得到自然对流和强追对流的边界层变 化的全息图，见图 2. 将上述变化现象用显微摄影或录象实时记录下来,最后进行计量处理。

在自然对流的状态下，作者测定了 KDP 晶体柱面距 [010] $2 \mathrm{~mm}$ 处，溶质边界层的厚度 8 。随过饱和度不同而改变的结果,见图 3 .

\section{2. 结果与讨论}

1) 溶质边界层的厚度 $\delta_{c}$ 由图 3 可见,在自然对流的状态下， $\delta_{c}$ 值随过饱和度的增加 而线性增加。这一实验结果与 Nernst 扩散理论方程基本一致。在静止的溶液中 Nernst 扩 散层厚度 8 与过饱和度成正比关系，从这一角度来看，溶质边界层也就是扩散边界层. 然而 实验还表明：在同一晶体的不同晶面和同一晶面的不同位置上，边界层厚度各处是不等的。 如图 $2 \mathrm{a}$, 在离 $[010]$ 为 $5 \mathrm{~mm}$ 处, 锥面 $\delta_{\mathrm{c}}-99.3 \mu \mathrm{m}$, 柱面 $\delta_{c}=230.9 \mu \mathrm{m}$. 而在 $10 \mathrm{~mm}$ 的位置 

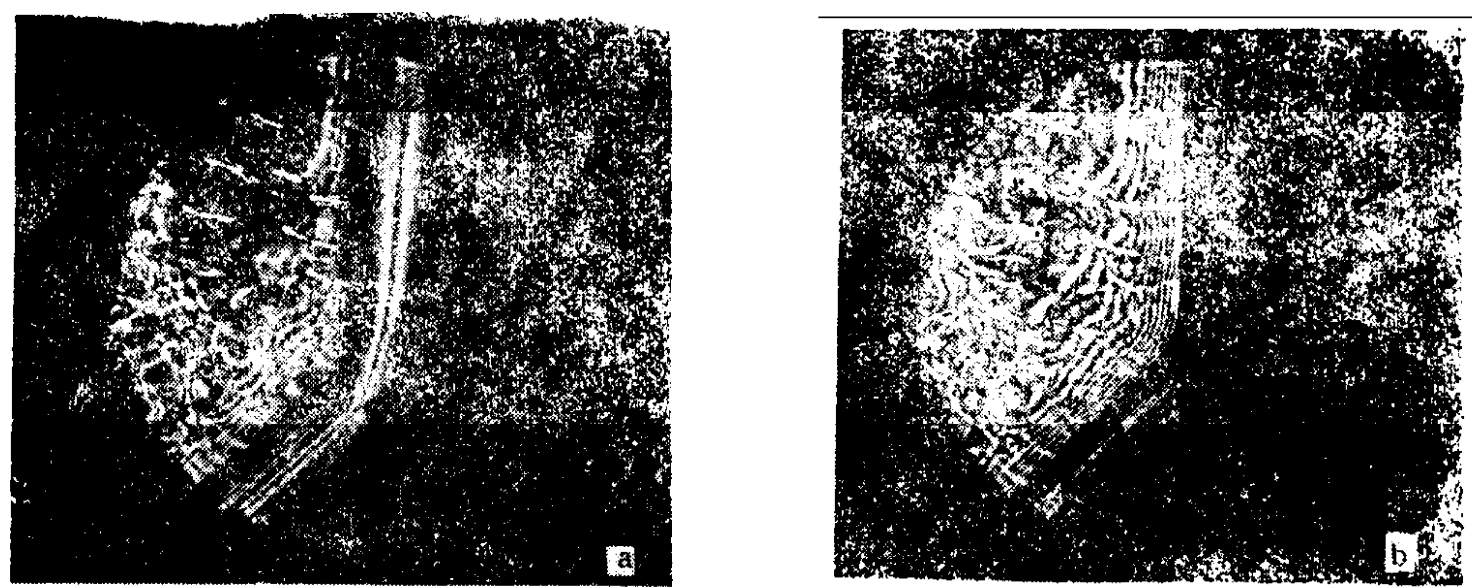

图 $2 \mathrm{RDP}$ 晶体,生长温度 $29.05^{\circ} \mathrm{C}, \Delta C=1.02 \%$ 时, 浴液对流状态不同的全息图

a. 自然对流; b. 强迫对流

上,柱面 $\delta_{e}-286.8 \mu \mathrm{m}$. 根据 Nernst 扩散方程,在同一柱面的上述等同面积上 $\delta$ 浽相等，然 而实验却表现出不等. 这就说明: 在重力场的作用下, 自然对流的影响是不可忽视的，因而只考虑扩散作用 是不够的. 实际上,在边界层内,不仅存在着分子的扩 散,而且还存在着对流作用. 对流不仅横穿边界层,还 有顺沿边界层的分量.

2）边界层内的浓度分布实验记录的是光波 位相变化的干涉条纹, 位相的改变是由于边界层内溶 质浓度变化引起的、干涉条纹的轨迹由溶液的折射率 变化而定,其关系为

$$
\Delta n=\frac{(2 K+1)}{2 d} \lambda,
$$

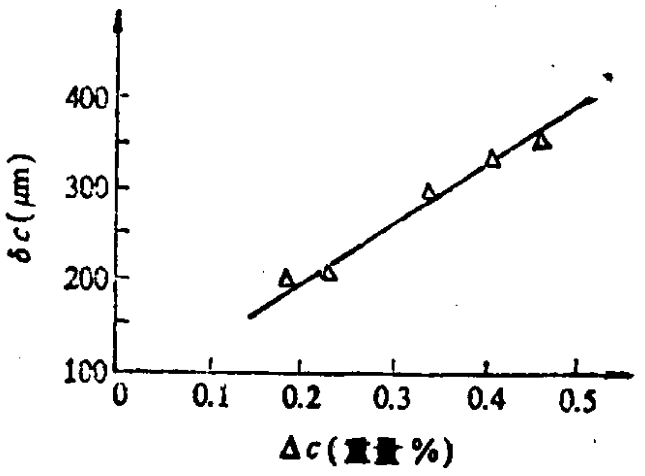

图 3 KDP 晶体柱面, 浴质边界层的F 度 $\delta_{c}$ 与过饱和度 $\Delta C$ 的关系

式中 $\Delta n$ 为折射率改变, $\lambda$ 为光波波长, $d$ 为光通过位相物体的厚度.

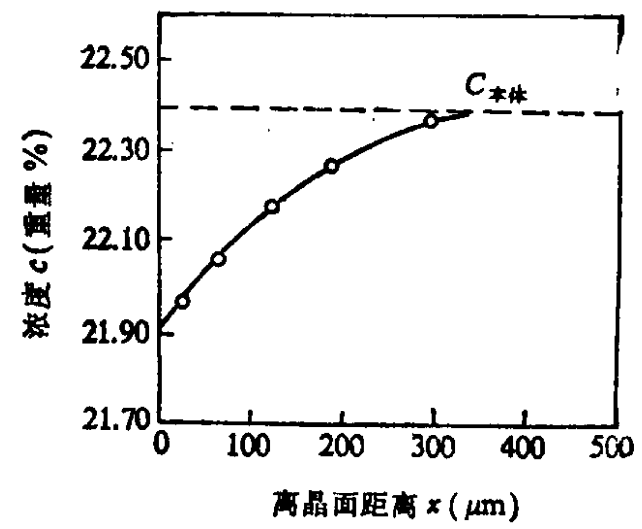

(a)

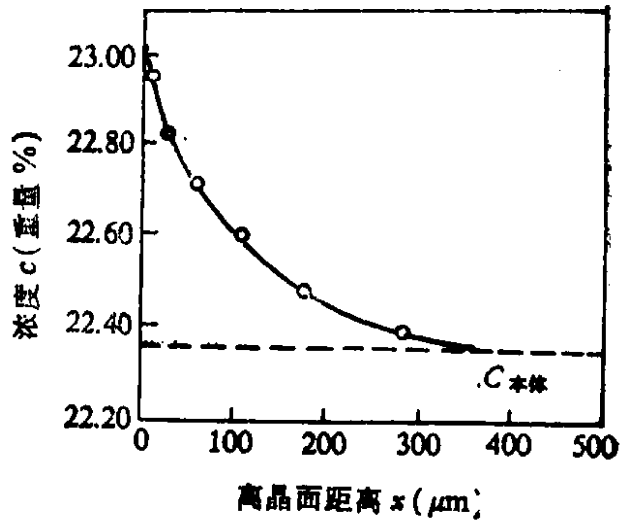

(b)

图 $4 \mathrm{KDP}$ 溶液在自然对流下,边界层内浓度分价曲线

a. 昆体生长 $\Delta C=1.04 \% ;$ b. 溶解 $\Delta C=-0.19 \%$

当实验温度为 $29^{\circ} \mathrm{C}$ 时, KDP 溶液的折射率与浓度的关系式为 ${ }^{[7]}$

$$
n=1.3304+0.00113 C \text {. }
$$


测量每根条纹到晶面的距离,则第 $i$ 根条纹的溶液浓度为

$$
C_{i}-c-\frac{(2 i-1) \lambda}{2 d}\left(\frac{\partial c}{\partial n}\right)_{T},
$$

式中 $C$ 为溶液浓度, $n$ 为其折射率, $T$ 为温度。

例如，图 1a 中在距 [010] 5mm 处的锥面上浓度分布曲线为图 4a. 图 4b 为溶解时边界层 内的浓度分布曲线。图 4 表明: 晶体无论是生长还是溶解，边界层的浓度分布曲线均是非线 性的. 在自然对流状态下，层内浓度梯度 $d c / d x$ 的结束是缓慢的，尤其在过饱和度小的情况 下,这种现象更为明显。这与偏振过滤光干步计量结果 ${ }^{[8]}$ 是一致的,而不象文献 [9] 所观察的 $d c / d x$ 或 $d c / d y$ 在边界层的边缘迅速下降为零。该文献采用纹影法测量边界层厚度，这种方 法本身是不能做定量研究用的. 测量结果容易导致错误结论.

3）强迫对流对边界层的影响 由自然对流和强迫对流的对比可见(图 2)，当过饱和度 均为 $1.02 \%$ 的条件下, 溶液在强迫对流时 $(50 \mathrm{r} / \mathrm{min})$, 晶体柱面 $\delta_{c}$ 迅速减薄接近零, 而雉面 $\delta$ 。由 $99.3 \mu \mathrm{m}$ 减薄至 $42.5 \mu \mathrm{m}$ 。边界层内的溶质浓度、速度与温度互相耦合构成一非线性函 数.

\section{四、结论}

1. 全息相村干涉显微术是相村显微术与全息实时干涉计量相结合的技术。它把两者的功 能灵活地结合在一起,不仅能深刻显示晶体生长过程的表面形貌和边界层的特性,还能在不同 液流效应及各种复杂过程中进行动态实时定量计量。该技术的优点是: 比经典干涉术应用范 围广;比全息相祄显微术易于定量化;比全息干涉术精确度高,直观方便。

2. 实验证明: 在自然对流状态下, 晶一液界面的溶质边界层厚度, 随溶液过饱和度的增大 而线性的增加. 边界层内的浓度分布呈非线性变化。

\section{考文蚌}

[1] Гусева, И. Н., Гинзбург, В. М., Крамаренко, В. А., Рост Кристаллов, XI (1975), 216-220.

[2] Petrovsky, V. A. et al., J. Crystal Growth, 56 (1982), 7-14.

[3] Kuijvenhoven, L. J. and Risseeuw, 1. J., Int. Sugar-Jnl., 85 (1983), 1010; 35-38.

[1] Bedarida, F., Boccacci, P., Zefiro, L., Physico Chamical Hydrodynamics, 6 (1985), 329-338.

[5] Yu Xiling and Wei Aijian, J. Crystal Growth, 83(1987), 11-14.

[6] 金铜、于锡玲,人工晶体, 17(1988), 123-127.

[7] 于铄、郡宗书、王瑞华,高等学校化学学报, $9(1988), 1252-1256$.

[8] Stanislas Goldsztaub, Roland Itti et al., J. Crystal Growth, 6 (1970), 130-134.

[9] Van Eackevort, W. J. P., Matuchova, M., Crystel Research Technology, 22 (1987), 167-176. 